\title{
DESIGN AND DEVELOPMENT BRAIN INSPIRED INTELLIGENT CONTROLLER FOR PMSM DRIVE
}

\author{
MD Qutubuddin ${ }^{1} \&$ Yadaiah $\mathrm{N}^{2}$
}

\begin{abstract}
This paper proposes a brain inspired intelligent speed controller for electrical drives to overcome the complex issues due to nonlinear nature of its winding currents and speed. The proposed control strategy is developed using limbic system of mammalian brain and called as Brain emotional controller. This controller is applied for many applications to achieve fast and good results in non-linear applications of control system. The brain emotional controller is developed as speed controller for Permanent Magnet Synchronous Motor (PMSM) drive. The effectiveness of proposed controller performance is analyzed for different speeds. The results gives that brain emotional controller characteristics are robust and fast response with respect to change in motor parameters, load and speed. The proposed system enables the designer to use for multiple objects of their choice.
\end{abstract}

Keywords: Speed, Torque, Currents, mammalian brain, amygdala, Orbitofrontal cortex (OFC)

\section{INTRODUCTION}

Now-a-days industrial applications of the PMSM drives are increased because of its advantageous features like high torque to inertia ratio, high power density, and high speed operation compared to other conventional ac motors, low noise and higher efficiency. In spite of this features PMSM machine requires a precise controller due to nonlinear nature of winding currents and speed. There has been a significant advancement in the development in the controlling of electrical drives. The d-q transformation applicable to the electrical drives can be considered for feedback linearization transformation. The recent developments in control theory of electrical drives made control engineers to develop a controller not only consider the nonlinearity of the system and also develop the controller which is not used previously. This becomes a challenge for researchers to develop a precise controller. Many researchers have made an attempt to make a precise controller to achieve better results compared to the conventional controllers.

In this paper, it is proposed a new kind of intelligent controller which is based on the emotional process of the mammalian brain i.e. Limbic system; this type of controller is called Brain Emotional Learning Based Intelligent Controller (BELBIC) [1-2]. Designing of controller is simple and unique, overcome the problems associated with conventional controllers. This controller is independent of system parameters, quick auto learning and proper tracking reference adaptability is achieved, which gives better performance.

\section{MODELING OF PMSM DRIVE}

The mathematical model of PMSM drive is described by the following equations and assumptions in a synchronously rotating d-q reference frame [3-5].

$\left[\begin{array}{c}V_{q} \\ V_{d}\end{array}\right]=\left[\begin{array}{cc}R_{q}+L_{q} P & \omega_{r} L_{d} \\ -\omega_{r} L_{q} & R_{d}+L_{d} P\end{array}\right]\left[\begin{array}{c}I_{q} \\ I_{d}\end{array}\right]+\left[\begin{array}{c}P \omega_{r} \psi_{f} \\ 0\end{array}\right]$

$T_{e}=\frac{3 P}{2}\left[\psi_{f} I_{q}+\left(L_{d}-L_{q}\right) I_{d} I_{q}\right]$

$T_{e}=T_{L}+J_{m} P \omega_{r}+B_{m} \omega_{r}$

The PMSM drive when it is feeding from the supply mains, the required starting torque is provided from squirrel cage rotor winding. There are two types of starting process of the PMSM drive, which are based on the mode of drive operation namely, 1) unsymmetrical asynchronous motor mode and 2) permanent-magnet-excited synchronous generator mode. If one wants to try the drive to be operated, start or run up to the synchronization, the effects of the shorted rotor windings are considered. However, the PMSM model in equations in (1) to (3) does not describe the asynchronous behavior of drive, since the motor is fed from variable frequency inverter. The PMSM drive can be operated up to the wide range of temperature by using modern magnets like NdBFe. Within the normal operating range of temperature the residual flux density and intrinsic coercivity will not affect, it has shown that it will decreases as the temperature get increased. These values can attain their previous or original values as the temperature comes down and reaches to its normal value. The dynamic behavior of the

${ }^{1}$ Dept. of EEE, JNTUH College of Engineering, Hyderabad, India

${ }^{2}$ Dept. of EEE, JNTUH College of Engineering, Hyderabad, India 
controller is affected by the variation of residual flux along with the stator resistance. In the normal operation, PMSM owes to the saturation, which will lead to the unsatisfactory performance of PMSM drive due to the linearization of PMSM constant control parameters, with standard d-q axis PMSM model. By adjusting the motor control parameters in accord with the changing saturation conditions, the improved prediction performance of the drive can be accomplished.

\section{COMPUTATIONAL MODEL OF EMOTIONAL CONTROLLER}

On successful implementation of the emotional controller in control engineering applications it is motivated to implement in the same in the electrical drives. The emotional controller is based on the of behavior of the limbic system of the mammalian brain, it is first implemented in solving SISO systems and extended to MIMO systems to solve the nonlinearity associated in the system and named as Brain Emotion learning based Intelligent Controller (BELBIC). In the proposed system Moren and Balkenius [1] network model has been adopted, in which the computational model consists of the Amygdala, Orbitofrontal cortex, Thalamus and sensory cortex. These are the parts of limbic system of mammalian brain, which are responsible for emotions. The emotions for cognitive and intelligent can be controlled by two approaches, (1) Direct Control approach and (2) Indirect control approach. The proposed, Emotional Controller comes under direct approach.

The proposed model structure is based on the limbic system of mammalian brain. Mammalian brain and its parts is shown in Fig 1. In the proposed controller there is a separate learning process for the Amygdala, Orbitofrontal cortex and thalamus, this technique is based on the mechanism generation of sensory inputs and emotional cues. The generation of emotional output is based on the learning of amygdala. It has connections with other parts, which are responsible for generation emotional response.

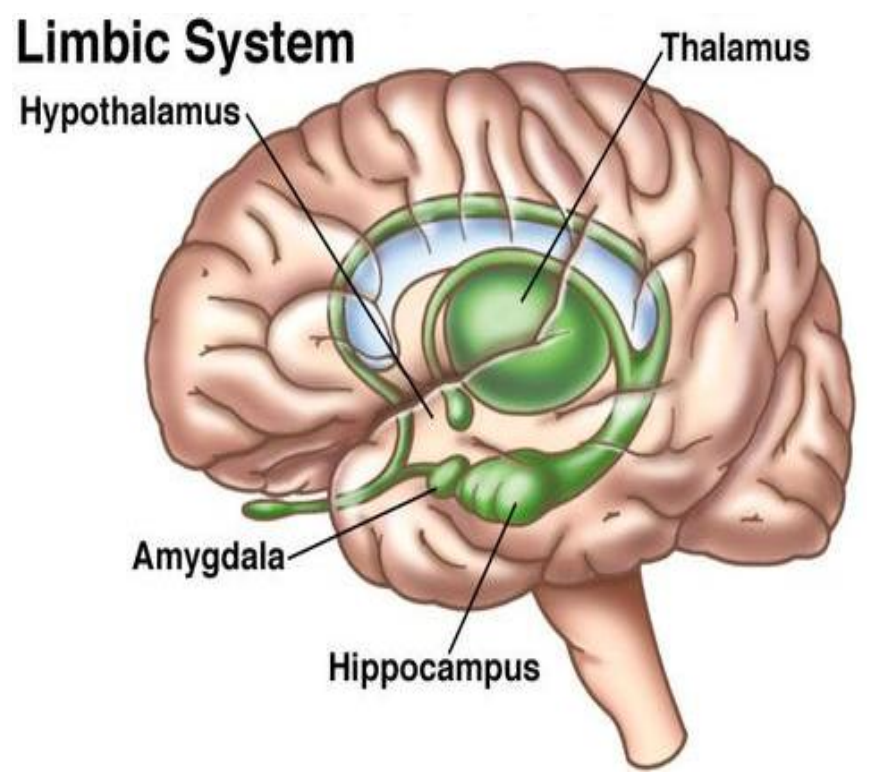

Fig.1 Parts of brain with limbic system

Fig 2 shows the computational model of the Emotional controller, there is A node for every stimulus $\mathrm{S}$ including thalamus connection. There is a $\mathrm{O}$ node for every stimulus $\mathrm{S}$ except Thalamus connection. The output of the system is denoted as $\mathrm{E}$ which is sum of all amygdala output subtracts from Orbitofrontal cortex output.

$\mathrm{E}=\sum A_{i}-\sum O_{i}$

Where $A_{i}$ is the amygdale output, which is derived as

$\mathrm{A}_{\mathrm{i}}=\mathrm{V}_{\mathrm{i}} \mathrm{S}_{\mathrm{i}}$

$\Delta V_{i}=\alpha \max (0, E C-A)$

Where $\mathrm{V}_{\mathrm{i}}$ is the gain of amygdala in connection, $\alpha$ is the learning rate of the amygdala, EC is the value of emotional cue function and A is value of amygdala's output at each time. The max term in equation (10) is for making the learning changes monotonic, implying that the amygdala gain can never be decreased.

Similarly orbitofrontal cortex output is given by

$\mathrm{O}_{\mathrm{i}}=\mathrm{W}_{\mathrm{i}} \mathrm{S}_{\mathrm{i}}$

$\Delta W i=\beta(\mathrm{MO}-\mathrm{EC}) \mathrm{S}_{\mathrm{i}}$

Where $\mathrm{W}_{\mathrm{i}}$ is the gain of OFC in connection, $\beta$ is the learning rate of the OFC, MO is the model output which difference of the amygdala and OFC and EC is the emotional cue.

$\mathrm{MO}=\mathrm{A}-\mathrm{O}$

Where, A - Amygdala output, $\mathrm{O}$ - Orbitofrontal cortex output 


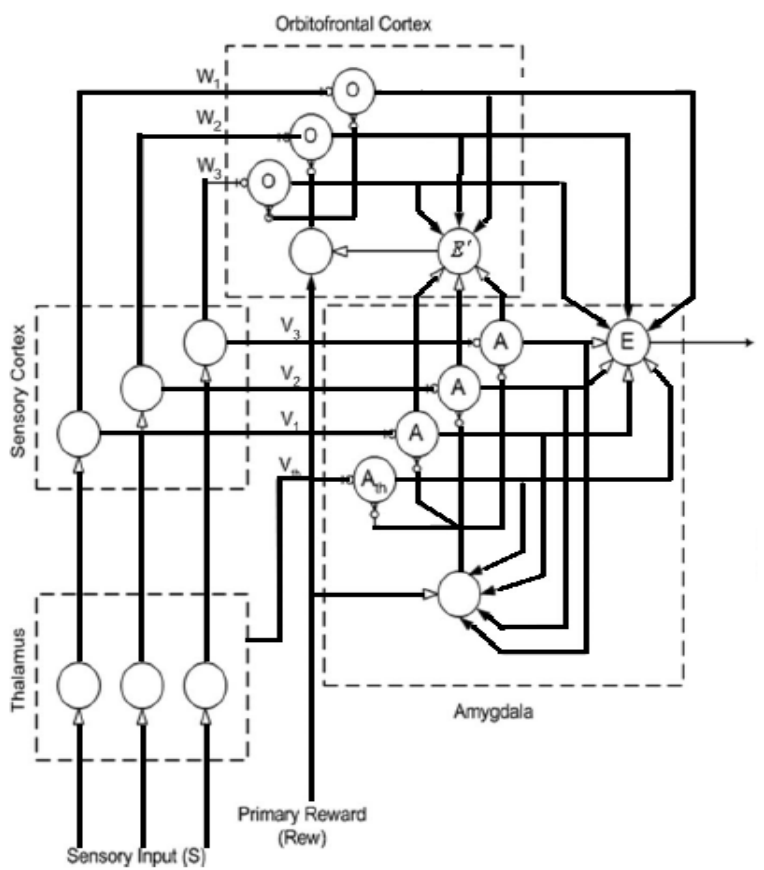

Fig.2 Computational model of the Emotional Controller

\section{IMPLEMENTATION OF EMOTIONAL CONTROLLER FOR PMSM DRIVE}

The fig. 3 shows the block diagram of PMSM drive incorporating of Emotional controller. The emotional controller works on the based on the receiving of error signal i.e. difference between the commanded value and the system generated value. In this work speed signal taken as reference, its commanded value and the generated value difference is given as input to controller. Accordingly the controller generates output i.e. the value of Iq with the assumption of $\mathrm{Id}=0$. The machine model is in the rotating reference frame oriented to the rotor flux and then are transferred to stationary reference frame, and by using $I_{d}$ and pre-calculated $I_{q}$ value, inverse Park's transformation is used to generate commanded a-b-c phase currents $i_{a}{ }^{*}, i_{b}{ }^{*}$ and $i_{c}{ }^{*}$. Hysteresis current controller is used to implement the Vector Control algorithm. To tune the inveter switches, Hysteresis current controller generates the logic signals, where these are generated by comparing the command current with the actual phase currents of the motor.

In the above said procedure, the controller controls the plant PMSM drive without any requirement of other conventional controller and the generated control variables are independent of motor parameters. This is makes an important factor for the designing the controller for power drive plants.

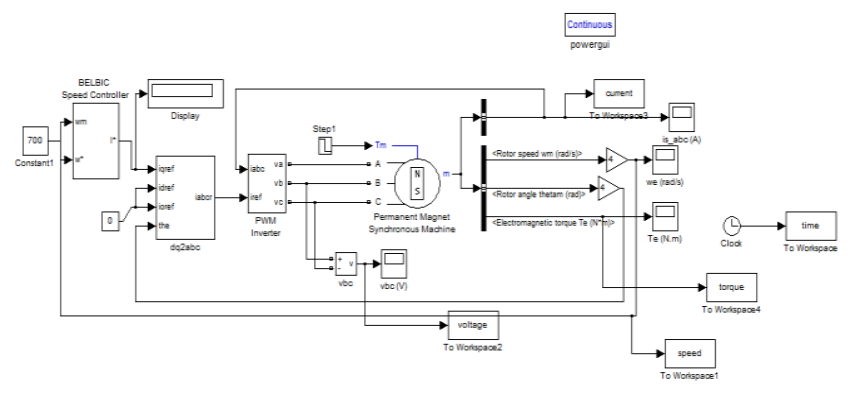

Fig.3 Simulation diagram of PMSM drive with Emotional Controller

\section{RESULTS AND DISCUSSIONS}

In order to verify the performance evaluation of proposed emotional controller for PMSM drive at different operating conditions and scrutinize the performance computer based simulations carried out in Matlab/Simulink at different speed commands. Accordingly, observed the current variations at different set of load and speed conditions.

The simulation results of PMSM drive with Emotional controller is observed for speed of $\mathrm{Wm}=700 \mathrm{rad} / \mathrm{s}$, and variable speed with different speed settings. The speed is tracked at $\mathrm{Ts}=0.02$ secs. With the set corresponding speed the winding currents and voltages are observed in Fig. 4, Fig.5 and Fig.6 respecitvely.. 


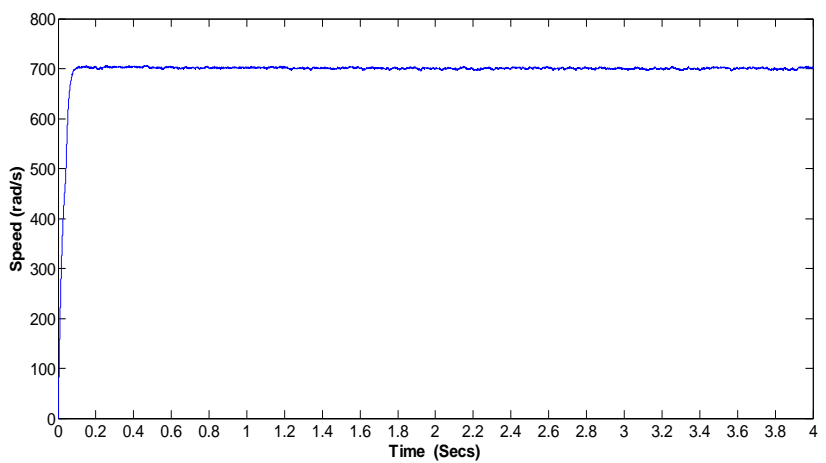

Fig.4 Speed response of PMSM drive

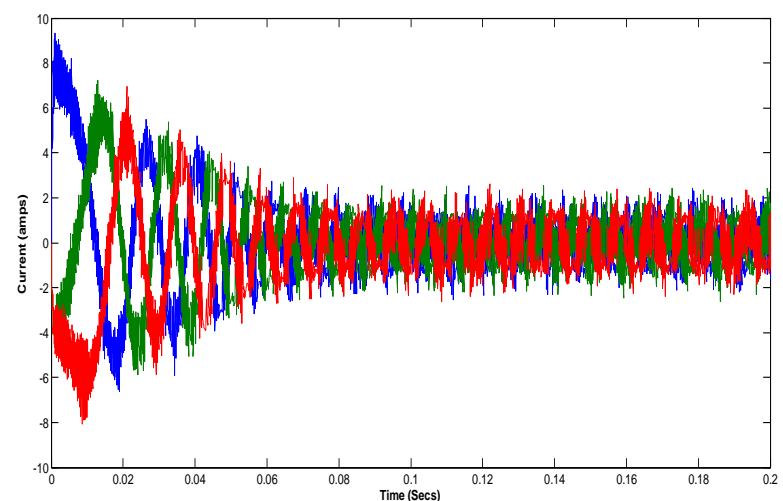

Fig. 5 Stator winding currents of PMSM drive

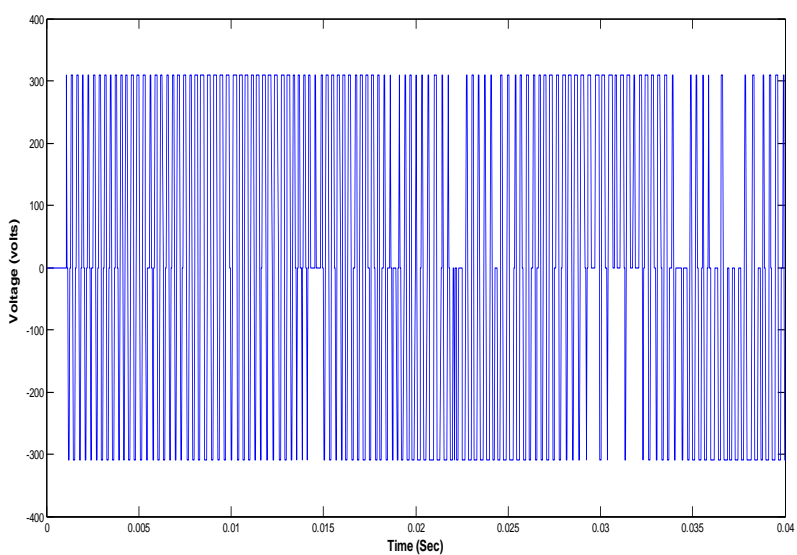

Fig. 6 Voltage waveform of PMSM drive

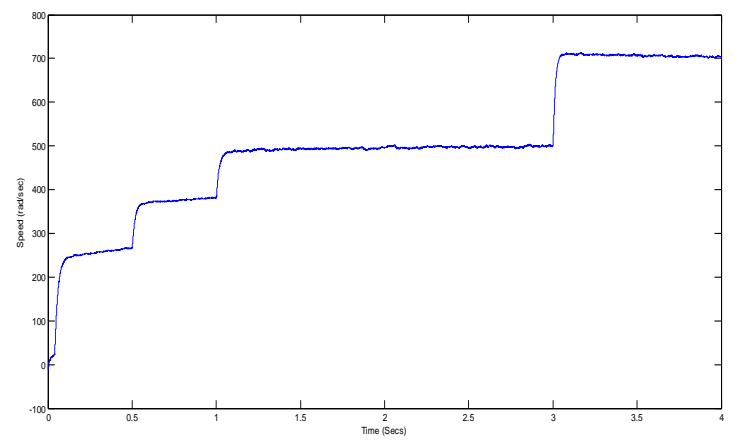

Fig. 7 Speed response of PMSM drive at different speed settings 


\section{CONCLUSIONS}

In this paper a new technique Emotional controller for PMSM drive has been presented with Matlab simulation. The controller is based on the behaviour of the Limbic system of mammalian brain. The proposed emotional controller contains six gains for the Sensory input and Emotional Cue apart from the gains of the Amygdala and Orbitofrontal Cortex. This makes the proposed controller effective and flexible for high speed applications.

\section{REFERENCES}

[1] J. Moren, C. Balkenius, "A Computational Model of Emotional Learning in The Amygdala: From animals to", in Proc. $6^{\text {th }}$ nternational Conference on simulation of adaptive behavior, Cambridge, Mass., The MIT Press, 2000.

[2] M. Fatourechi, C. Lucas, A. Khaki Sedigh "Reducing Control Effort by means of Emotional Learning" Proceedings of 9th Iranian Conference on Electrical Engineering, (ICEE200J), pp: 41-1 to 4 1-8, Tehran, Iran, May 2001.

[3] .M. N. Uddin and M. A. Rahman, " Fuzzy Logic Based Speed Control of an IPM Synchronous Motor Drive", Journal of Advanced Computational Intelligence, Vol. 4, No.3, 2000, pp. 212-219.

[4] T. S. Radwan, M. A. Rahman, A. M. Osheiba, and A. E. Lashine, "Dynamic analysis of a high performance permanent magnet synchronous motor drive," Proceedings Canadian Conference in Electrical and Computer Engineering, pp. 611 614, 1996.

[5] P. H. Mellor, F. B. Chaaban, and K. J. Binns, "Estimation of parameters and performance of rare-earth permanent-magnet motors avoiding measurement of load angle," Proceedings, Institution of Electrical Engineers, vol. 133, pp: 322-330, November 1991. 